\title{
Automating the Drug Dosage of Tacrolimus for Liver, Renal Transplant Patients using Neural Network
}

\author{
Nijitha Thomas K., Aswathy Wilson
}

\begin{abstract}
Nowadays in medical field the major concern lies in the field of liver, renal diseases. Liver is the largest organ in the body and it is the factory which processes all the foods we taken. We should keep liver in perfect condition. But today there were lot of Liver, renal damages occurred commonly. where sluggish lifestyle of humans and escalated alcohol abuse has become dangerously common, liver, kidney health have regained focus. This can cause liver cirrhosis and liver dysfunction. The main solution for this is transplantation surgeries. In most of the cases, transplantation surgeries are successful. But after few days normal patients become die. its a very common news. This is because of the lack of ideal drug dosage prediction. Today all of the medical practitioners calculate manually using some patients responses towards the drug. So it is not a systematic approach. Only purely mathematical approach is available for calculating drug dosage.

To achieve an optimal drug dosage calculation, proposed model will automate this system based on some patients response data like cell viability, drug trough level, Creatine Test result, biopsy result, MELD score etc using some artificial intelligence techniques like neural networks. The human and monetary of both optimal and Sub-optimal drug dosage may be deduced from the action of various optimized neural networks. Neural networks provide sceptical help to doctors. Currently there is no system will automize this dosage calculation. This calculation based on patients responses after transplantation surgery. Normally start with zero level dosage of medicines. After few days the ideal drug usage calculations occurred based on some observing patients different levels of data. Automate this system will help to doctors to calculate automatically the optimal usage of drugs makes precise calculations in the patients health.
\end{abstract}

Keywords - Artificial Intelligence, Artificial Neural Network, MELD score, Tacrolimus

\section{INTRODUCTION}

In medical field, there were lot of medical procedures implemented with the help of computer applications nowadays. Today it's necessary to keep all detailed patient's data using computer. Many of the techniques developed for medical imaging (CT scan, MRI scan) implemented using computer technologies. Robotic technologies (including surgical robots and rehabilitation robots) are widely used for many of the surgical procedures. The use of computer applications range in health care will be widely increased. Currently the application of computer technologies are widely used in drug therapy also. In this the major experiments will concentrate on optimal drug dosage calculation.

Revised Manuscript Received on December 15, 2019.

Nijitha Thomas K., M.tech, Department of Computer Science, Jyothi Engineering College Cheruthuruthy, Thrissur, Kerala, India.

Aswathy Wilson, Assistant Professor, Department of Computer Science, Jyothi Engineering College Cheruthuruthy, Thrissur, Kerala, India.
Because Inter variability and individual variability in requirements of dosage formally use guided by physicians quantified drug management, which gives in rampant variations from the objective dosage ranges.[12] Normally once a peculiar drug is chosen, the pharmacokinetics clinical principles are required to assure the pertinent management of drug is chosen for an administration appropriate route . On the basis of the patient's handling parameters of drug, which require a considerate of one basic pharmacokinetics metabolism and excretion, absorption, distribution, the regimen of dosage for the medicine in a appropriate patient can be developed. It is necessary to ensure that the appropriate regimen is prescribed to achieve optimal adequacy and nominal toxicity. Nowadays there were lot of liver and kidney diseases widely increased. The new lifestyle of the peoples were highly influenced these type of chronic diseases. Some of the diseases which will lead to the transplantation of liver or kidney.[1]

These are:

\section{viral hepatitis}

Alcoholic liver disease

Autoimmune hepatitis

Acute liver failure

Autoimmune diseases, such as lupus and $\operatorname{IgA}$ nephropathy

Nephrotic syndrome

Urinary tract problems

In these liver, kidney failures, the only solution for this is liver, kidney transplantation. Here the major reason lies in the lifestyle change

nd misconceptions about health in between people. where sluggish lifestyle of humans and escalated alcohol abuse has also the reasons for this kind of liver, kidney damages[2].

Transplantation is the procedure of the deportation of tissue from one part of the body or from one individual and its implantation or insertion in another. Most of the organs will be transplanted nowadays. Transplantation have vital role in survival of patients. In case of transplantation, tacrolimus is the main drug used for suppressing the immunity. For every person receives an organ or tissue from someone else during surgery of transplantation, that person's immunity system may observe that it is foreign. It is because of the person's immune system distinguish that the antigens on the cells of the organ are different. Mismatched organs, or tissues that are not matched intently enough, can generate a blood exchange reaction or transplant rejection. 
To avert this we used tacrolimus drug to allogeneic organ transplant to lower the risk of organ rejection. Tacrolimus have limited therapeutic dimensions. [3] It is mainly used representative, however, described by limited curative indicator and large interchangeability and individual changeability in stipulation of dose constraining incessant monitoring of curative drug to prevent rejection or kidney, liver toxicity. Many elements such as age, ethnicity, bodyweight, , haematocrit, gender, albumin, serum creatinine days after surgery of transplantation and the main usage of adjuvant drugs will highly clout the Tacrolimus pharmacokinetics. Not only that but also the genetic disparateness in ABCB1 and CYP3A5 affecting combination of tacrolimus as well and its scientific conclusion. CYP3A5_1 was the primitive- type allele is correlated with action of enzyme, while CYP3A5_3 was the major familiar allele it makes source graft shortage and concluding that non-utilitarian protein and they craving a tacrolimus high dose for the patients . Many studies have investigated the association of CYP3A5_1/_3 curative power and pharmacokinetics of Tacrolimus in transplantation surgery of kidney. The curative role in drug conveyor, ABCB1 gene on absorptions of trough of tacrolimus harvested blended results. One study characterized the corporation of ABCB1 polymorphisms with the adjusted dose tacrolimus trough concentrations during the initial period after transplantation [4]. The present apparatus generally matters the planning, training and use of neural networks especially deep neural network for the optimization of the authority of drugs in respect of characteristics of patients.

The modernization absolutely steps by step archives the new stuffs before we make use in comfort. The new cybernated answering innovation permits only takes few particular parameters to enhance the optimized drug dosage of tacrolimus .

ANN is a computational model that undertaking to represent the provisional idea of the human mind. An ANN is a system of enormously interconnecting handling components(neurons) working in comparative. The interconnections between components like neurons generally decide the system work. Layer in the neural network system is the subgroup of the manipulation component. The input layer is the initial layer after final layer is output layer. Between the input and output layer, there might be extra layers of units, called hidden layer. Increasing in the number of hidden layers increases the efficiency of the system. where artificial neurons catch in a set of weighted inputs and produce an output over an activation function[6].

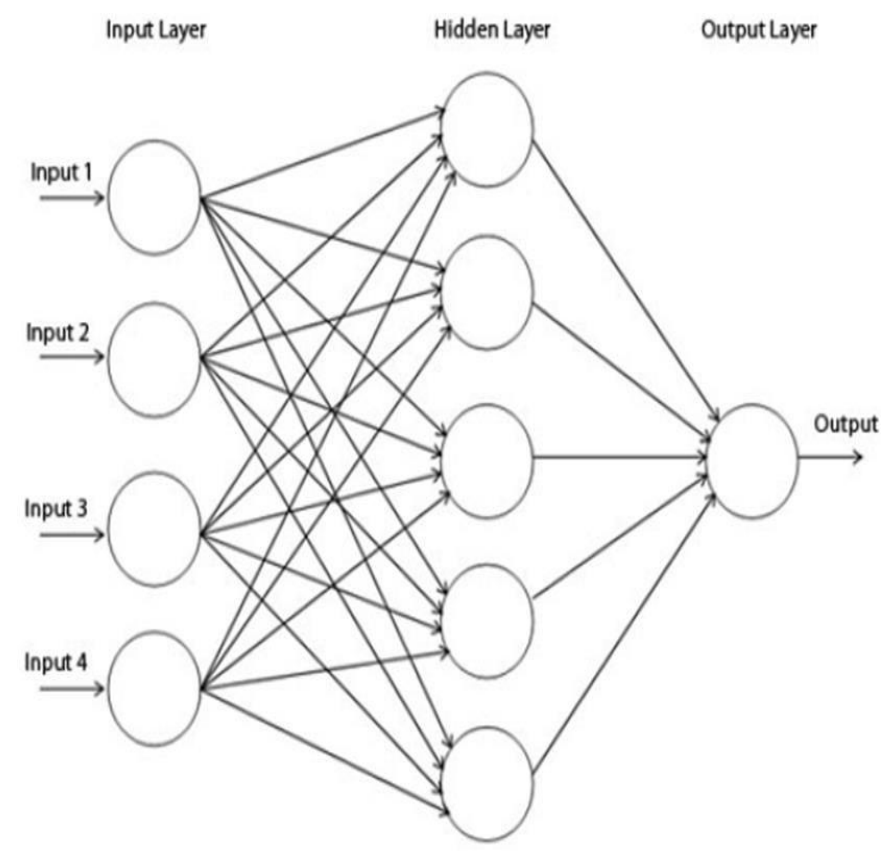

The complicated small multilayer systems were detached into two training allocations. They are named as supervised or administered learning and unsupervised learning . In handled pandemic in the system is adapted by allowing it information and harvest compositions. Among this moment, the neural system can have change the federated loads to correlative its harvest with the actual yield in an continual proceedings instil the point that an conventional result is come to.

Especially in medical field, the use of artificial intelligence(especially ANN) is widely increased. It will create more optimized calculations in the case of human health. The use of supervised learning techniques and unsupervised learning techniques will improvize the efficiency of calculations or predictions.

\section{RELATED WORK}

Individualizing liver transplant immune-suppression using a phenotypic personalized medicine platform[3].This system aims to improvise the efficiency of finding the trough ranges of tacrolimus using a parabolic personalized dosing platform. In which the drug consolidation represents an input apportionated by a doctor to the patient. Corresponding to the drug initial dose the patient then reacted with outputs which are based on phenotypic measures, such as the potency and/or morbidity of therapy[3] . It ascertained that the input as drug and output based on phenotypic relationship is represented by a second or high order polynomial equations, that is, a surface which is like a parabola . Here, the dosage level of tacrolimus was the "result"- which is calculated routinely as part of any patient's clinical health care; the input was only a one-drug Tacrolimus dose. This system mainly focused on the accurate trough ranges or bioavailability of tacrolimus drug by using purely mathematical concepts such as taylor series expansions. It is very complex in nature. In next Neural drug dose estimation [5] This is an another kind of system in which it is purely based on computational techniques. 
This system is mainly suited for mental health especially for psychotropic drugs. Here neural networks are developed (programmed), trained on training data, and used to predict or calculate any of optimal drug dosage of any drug rather than the protruded drug dosage. It is the developed system to find the

\section{- $\quad$ Single drug optimal dosage}

- Optimal dosage of one drug with respect to other drug

- Optimal drug dosage with respect to the patients characteristics

- Optimal drug finding in the basis of cost

It is developed for improvise the low therapeutic ranges of different psychotropic drugs.[5]

At the same time, for the procedure of selection, the best neural network trained on the historical trained patient clinical data. This training may be, in many ways , in accordance with either the (i) Levenberg Marquardt (L-M) algorithm or the (ii) back propagation methods of neural network optimization. The lack of data in medical field leads to use LM algorithm for better prediction availability. This system is very effective for mental retarded patients.

Next, Artificial neural network model for predicting the bioavailability of tacrolimus in patients with renal transplantation [4]. This system is mainly focused the effect of two genetic allels such as CYP3A5 and ABCB1 organic phenomenon in obtaining the dosage ranges of tacrolimus and the risk for post transplant surgery

patients diabetes. This system is used artificial intelligence techniques and Logistic regression techniques to model the drug dosage predictor. It used error-correcting output coding or averaging by Bayesian formula , bagging and boosting as the basis of the model. The composed ANN architecture have three layers . first an input layer, a hidden layer and an output layer. The input variables used in this work as age, body mass index , gender, creatinine, CYP3A5_3 $(6986 \mathrm{~A}>\mathrm{G}), \quad \mathrm{ABCB} 12677 \quad \mathrm{G}>\mathrm{T} / \mathrm{C}, \mathrm{ABCB} 13435$ $\mathrm{C}>\mathrm{T}, \mathrm{ABCB} 11236 \mathrm{C}>\mathrm{T}$ and. The number of nodes in the hidden layer was optimized up to ten in this work using Root Mean Square Statistics method.[4]

All human service all arounders need to make the best outputs for each and every persons who have done by a transplantation. A doubtful access toward a normal life of a patient after the transplantation surgery is never anew advisable. The current communal allowance best persons aware that it is needful for all discplines to coordinate to bring out good results for patients by being proficient about right on time and forceful assessment and treatment proposals. Not only that but also in case of transplanted patients, the optimal drug dosage is the most important aspect.

\section{PROPOSED SYSTEM}

Most inspections performed on the automatised Inquiry of bio -availability of Tacrolimus drug for transplanted patients in the basis of some specific elements.. An artificial neural system allowing more clear-cut able outputs and establishin case of life of the post surgery transplant patients. To avert the contingent of elimination or infection of an organ

by equitable dosage calculation of tacrolimus, it choice

up the personalized order calculation neural system. An Artificial neural system based expectation of dose ranges of tacrolimus enhances based on some patients responses with higher consistency. The maintenance of

kidney hybridization cases that are on tacrolimus is highly challenged to the doctors expected to the cramped therapeutic ration and wider inter variability and- individual variability. Concentrations of Tacrolimus varies highly during the urgent post-

transplant time and may be the imaginative component for the enlarged measure of infection or rejection and graft loss. The proposed system makes the trough ranges of tacrolimus automatically in patient specific pattern. It will take more minute features effect on predicting the bio - availability of this drug.

\section{DATA COLLECTION}

The data can collect from the medical institutes and National Institute of Diabetes and Digestive and Kidney diseases(NIDDK). it also includes more than 30 features which contain, It includes

patient history, hospital details, risk factors and symptoms. Risk factors include Age, Gender, Blood Pressure, daily creatinine, MELD score, CYP3A5

_1/_1:_1/_3:_3/_3, MRI results, ABCB1 1236 CC:CT:TT, ABCB1 2677 GG:GA/GT:TT/AT/AA,

ABCB1 3435 CC:CT:TT, nationality, age, liver disease, disease severity, kidney function, comorbidities, concern for rejection, and use of other immune suppressants, BIOPSY test results, etc

\section{DATA PREPROCESSING}

This dataset is highly confronting to usage adequately due to a compelling portion of lossing values and large number features in this dataset. In this method eliminates the noisy, missing data, duplicate records, inconsistent data, and. Some data are missing should be removed from the database to improve the classification performance of the network. It is highly suitable features are carefully selection for finding drug dosage of Tacrolimus. The dataset lessens the unpredictability related to the classification.

\section{DATA ENSEMPLE TECHNIQUES}

This system will ensemble the data neural network model by using two techniques

Bagging

Boosting

Bagging: This is an ensemble technique in which the main principal goal is to make a group of weak learners combined together to form a strong learner. Bagging is the utilization of the procedure of Bootstrap to a high-deviation machine learning algorithms. Bagging is a method to cutback the deviation in the prediction by creating extra data for training from dataset using consolidation with repetitions to produce multiple -sets of the original data. [10] 
Boosting: It is an another ensemble technique which will be used in this system. It is an continual technique which accustoms the weight of an experiment based on the final classification. If an observation or an experiment was classified inaccurately, it tries to boosting the weight of this observation. Boosting in general builds high strong predictive models[11].

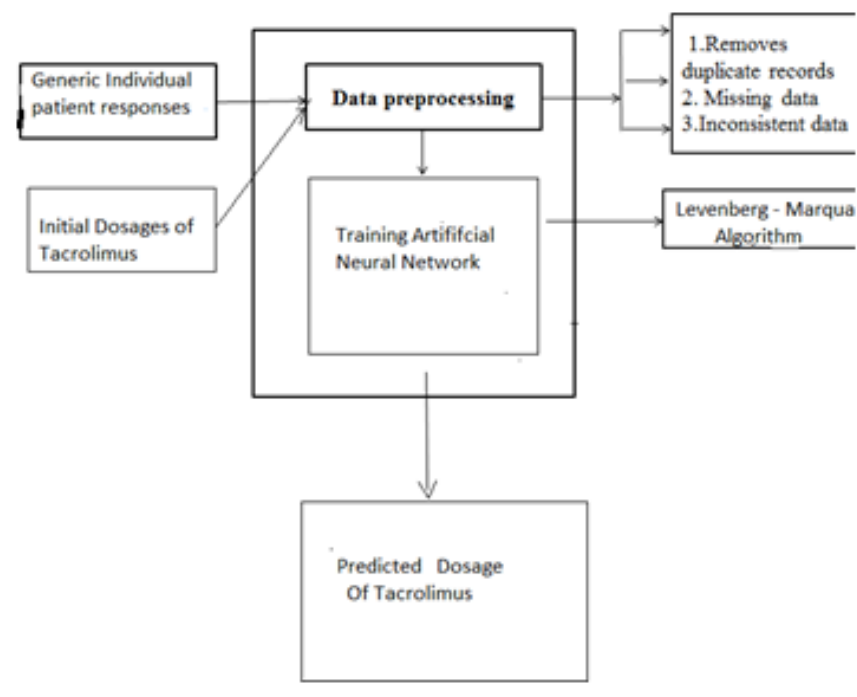

Fig 1: Architecture diagram for Finding the dosage of Tacrolimus

\section{ARTIFICIAL NEURAL MODELLING \\ NETWORK}

Now a day's most of the peoples have been using AI technique for many applications in the medical field. This system control gaining from a dataset with many levels of consideration by computational models that are associated with numerous layers. This method outlined to gain highly complex variables that are to be a part of the result.

In neural network, Each of the connections between the layers have a figure correlated with it called the associate weight and each individual neurons has a figure known as threshold value and an appropriate formula associated called activation function respectively. These are the main parameters of the neural network in artificial intelligence. The neural network is being trained by implemented with a group of inputs and their interrelating outputs. It calculates the inputs through the neurons on each of the layers of the network, using an activation function, and the output of each layer will forward on to the next layer and so on. Finally the result in the output layer compared the result with expected result and it checks how distant the two results and proportionately adapts the parameters or values on each of the neuron layers through LM ( Levenberg Marquardt) algorithm to bring the correct and produced outputs as close to each other as possible. This is the way the neural network were to be learned.

\section{- Levenberg - Marquardt Algorithm}

It is the neural network training algorithm used in this system. It is further familiar as damped least - squares method. It has been created to work categorically with functions of loss which catch the model of a sum of squared errors. It works mainly based on gradient descent method and Jacobian matrix. It does not take hessian matrix into account. This system takes LM algorithm for training the neural network. The limitation of the high availability of large dataset is to be a major issue. In these scenarios, the LM algorithm were more suitable to train the network [7].

After disparate aspect representations have been conferred to the network, the weighted parameters accordingly observe to a supervised feature. Developments of the previous layers or steps become input to the next layer or next step. The increased number of layers, will compute the faster model. The hidden layers can handle as dribbling the final output. However, it is required to decide another time whether the outputs are valid. When the patient details and initial dosage were entered, it checks through the trained model and predicts the chances of rejection or infection of an organ and also predict the corresponding personalized patient Tacrolimus drug trough ranges.

\section{EXPERIMENTAL RESULTS}

The use of trained neural nets in accordance with the present invention for Tacrolimus trough range prediction. The method is fundamentally agreeable to a large amount of patient interaction. The ANN simulations depicting genotype based association of tacrolimus bioavailability in men and women are comparatively high with doctors prescribed dosage immune suppression using a phenotypic personalized medicine platform.

\section{CONCLUSION}

In medical field, the recognition of major risk factors in the case of transplantation surgeries become very difficult. This system enhances the ability to overcome these risk factors by correctly predicting the dosage of Tacrolimus for transplant patients in a personalized manner. It will helps the doctors for efficient calculation and also can avoid acute rejection or infection of an organ. This predictive method will take minute features that will effect the variance in dosage of tacrolmus and maintain the corrected output dosage for the post transplant patients.

\section{REFERANCES}

[1] http://www.chp.edu/our- services/transplant/liver/liver-transplantnecessary/liver-diseases-

[2] https://transplant.surgery.ucsf.edu/conditions-- procedures/chronickidney-disease.aspx

[3] Ali Zarrinpar, Dong-Keun Lee, Aleidy Silva, Nakul Datta, Theodore Kee, Calvin Eriksen, "Individualizing liver transplant.

[4] Kalluri Thishya, Kiran Kumar Vattam, Shaik MohammadvNaushad, Shree Bhushan Raju, Vijay Kumar Kutala,v"Artificial neural network model for predicting thevbioavailability of tacrolimus in patients with renal transplantation"PLoS ONE 13(4): e0191921.

[5] Sharon S. Tang, Cornelius Diamond and Scott Arouh,, "NEURAL NETWORK DRUG DOSAGE ESTMATION", M. U.S. Patent US 6,658,396 B1, dec2, 2013.

[6] https://en.wikipedia.org/wiki/Artificial_neural_ne twork

[7] https://www.neuraldesigner.com/blog/5_algorithms_to_trai n_a_neural_network 
[12] Hua Xu1, PhD, Son Doan1, PhD, Kelly A. Birdwell2, MD, James D. Cowan5, Andrew J. Vincz2, David W. Haas2,3, MD, Melissa A. Basford4," An Automated Approach to Calculating the Daily Dose of Tacrolimus in Electronic Health Records"

[10] Kristína Machová, František Barčák, Peter Bednár," A Bagging Method using Decision Trees in the Role of Base Classifiers", Acta Polytechnica Hungarica, Vol. 3, No. 2, 2006. 